\title{
Personalized and Deformed Avatars for Realizing Avatar-Mediated Real Person-to-Person Communication
}

\author{
Masayuki Heike, Kinya Fujita, and Takahiro Tanaka \\ Tokyo University of Agriculture and Technology \\ 2-24-16 Nakacho, Koganei, 184-8588 Tokyo, Japan \\ \{50007646124ast, kfujita@cc, takat@cc\}.tuat.ac.jp
}

\begin{abstract}
In avatar-mediated communication, there is a potential risk of familiarity detraction caused by differences between the appearance of the user and the avatar. However, the personalized avatars did not produce familiarity against the expectation. In this study, deformation rules extracted independently of the aspects of the models are discussed by comparing seven cartoon portraits to the originals. An avatar personalizing tool based on the averaged deformation proportions was developed. It was experimentally confirmed that the personalized and deformed avatars produce more familiarity.
\end{abstract}

Keywords: Communication, Avatar, Avatar Personalization, Deformation.

\section{Introduction}

Popularization of broadband networks has spurred the growth of multi-party, realtime, on-line communication systems based on text, voice, and video information. Video-chat systems have the advantage of allowing users to recognize facial expressions and gestures of their partner, compared to other forms of communication systems. The communication quality of a video chat is therefore considered closer to real face-to-face communication than other forms of communication. However, the direct use of video images risks unintentionally giving out personal information. This privacy risk is more serious for personal users and hence limits the number of users. Avatar-mediated communication systems can provide a multi-party, on-line communication system for users concerned about privacy.

Human communication depends on both nonverbal and verbal information [1]. Nonverbal information is considered to provide as much as $93 \%$ of the information in face-to-face communications [2]. One potential disadvantage of avatar-mediated communication is the absence of this nonverbal information that is naturally expressed by users. The missing nonverbal information must be adequately compensated for in order to attain natural communication that is equivalent to face-to-face communication.

To compensate for the missing nonverbal information in avatars, many systems have introduced manual control functions for the behavior and appearance of the avatar. Some studies have been carried out to automatically compensate for the nonverbal information, such as nod control by detecting the end of the utterances [3], 
gaze control based on a human gaze targeting model and paralinguistic information [4]. Personalized avatars based on photo images that reflect the physical features of the user have also been studied. However, a simple personalization of the physical shape of the avatar does not produce familiarity [5]. This is similar to the "uncanny valley" problem [6], in which human observers offer a response of revulsion when robots and other facsimiles of humans look and act almost like real humans.

In multi-party communication systems, the avatars should produce a sense of familiarity in terms of making a positive impression and reflecting the features of the participants. In this study, we focused on the positive impressions of characters in Japanese cartoons. We extracted deformation rules from caricatures and averaged them. Impressions of the personalized and deformed avatars were subjectively evaluated. This paper discusses the extracted deformation rules and the effects of deformation and personalization on familiarity.

\section{Networked Voice Communications}

\subsection{Avatar-Mediated Real Person-to-Person Communication}

Real-time network communications are basically divided into two categories. The first is direct communication, shown in Fig. 1(a), such as video chatting. It allows users to imagine the communication partner and the partner's status from a displayed image. The second is indirect communication, shown in Fig. 1(b), such as avatarmediated chat using avatars that do not reflect personal features. The inconsistency between the avatar appearance and the communication partner prevents users from recognizing the displayed avatars as the person they are communicating with. It is widely observed that the users act as puppet masters and talk only about topics within the simulated metaverse. In this case, avatar-mediated communication is considered to be quite different from direct communication, particularly in communication between acquaintances.

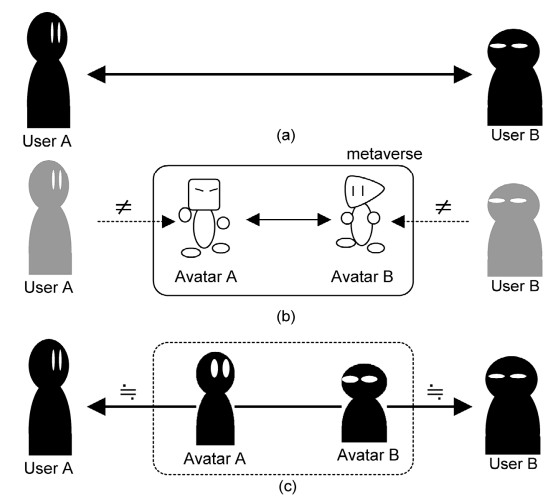

Fig. 1. Styles of networked communication and the consistency of appearance between the avatar and the speaker. (a) Direct communication such as a video chat. (b) Avatar-mediated communication using non-personalized avatars. (c) Avatar-mediated communication using personalized avatars. 
This study aims to realize yet another style of communication-avatar-mediated real person-to-person communication, shown in Fig. 1(c). Personalized avatars provide more consistent avatars for the speakers and should allow more direct communication between the users. At the same time, the avatars protect their privacy.

\subsection{Personalized Avatars with Familiarity}

A former study revealed that the personalized avatars, whose shapes are proportional to that of the actual user, provide more negative impressions [5]. This was thought to be due to the "uncanny valley" effect [6]. The enlargement of the eyes and the mouth was effective in improving the sense of similarity, not familiarity [5]. This is possibly due to the simplification of the shapes and textures that might weaken the impression of the facial features.

We propose a dual-axis model that includes similarity and favorability, to discuss the familiarity of avatars, shown in Fig. 2. Physically personalized avatars have a higher similarity to the person they represent, but are less favorable due to the "uncanny valley" problem. In contrast, well-designed cartoonish characters such as deformed animals have a higher favorability but no similarity. Neither of these two examples provides similarity and favorability at the same time. Therefore, we propose a cartoonish deformation of a personalized avatar to provide a balance between similarity and favorability. The compatibility of the two factors is expected to provide greater familiarity in communication between acquaintances.

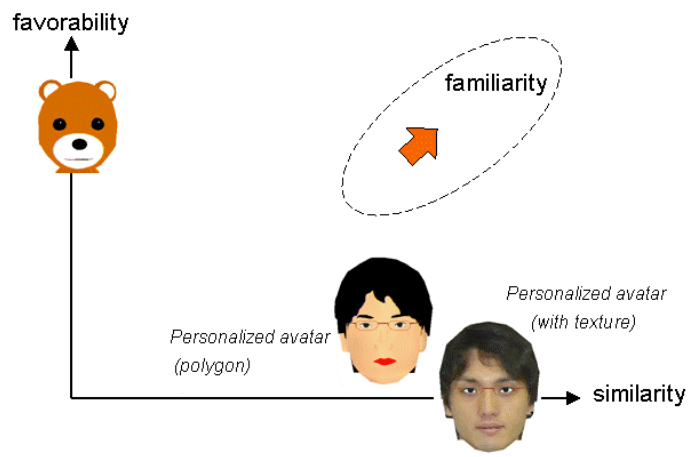

Fig. 2. Similarity-favorability model for discussing avatar familiarity

\section{Avatar Personalization Tool}

We developed an interactive avatar personalization tool that adapts the shape of the standard 3D head model to the shape of the user [5]. The head model has 398 vertices and 539 polygons. The personalization tool allows GUI shape adaptation using front and side view images of the model, as shown in Fig. 3. A three step adaptation process was adopted for faster personalization. The first step is the entire scaling and translation for general adaptation. The second step addresses individual facial part scaling and translation, such as eyes, eyebrows, nose, mouth, ears, and hair. The last 


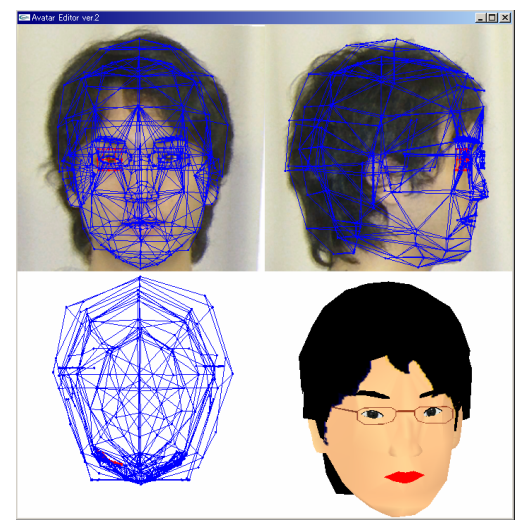

Fig. 3. Screenshot of the developed avatar personalization tool

is the adjustment of each vertex. In addition, the vertices for a smiling face were defined by adapting a customized normal face using the same tool for automatic facial expression control during voice chats based on paralinguistic information [4].

Automatic deformation is performed after the personalization process, based on a deformation rule that is extracted by the following procedure. It removes the necessity for the user to make subjective judgment and manual operation.

\section{Extraction of Standard Deformation Rules}

\subsection{Deformation Tendencies in Cartoonish Portraits}

First, we compared seven personalized avatars based on photo images and cartoonish portraits of the same persons drawn by an amateur illustrator, as shown in Fig. 4. Two common tendencies, 1) downward translation of eyes, eyebrows, nose, and mouth, and 2) enlargement of the features were observed.

Deformation might be considered an emphasis of personal features. However, there is a potential risk that arbitrary emphasis of personal features does not provide a favorable impression. Furthermore, generalized deformation rules simplify the deformation

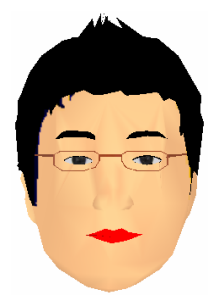

(a) personalized avatar

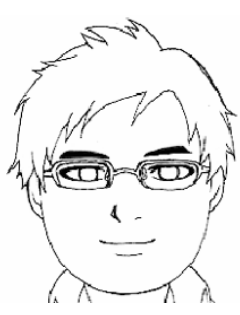

(b) cartoonish portrait

Fig. 4. Comparison of personalized avatar and illustrated cartoonish portrait 
process, and even the same deformation rule emphasizes differences in the shapes of the users. Therefore, the possibility of a general deformation rule is experimentally tested in this study.

\subsection{Extraction of Deformation Rules in Portraits}

According to the observed deformation tendencies, the downward translation, and the enlargement of the facial features such as the eyes and nose are modeled in eq. (1) and (2). The defined parameters are as follows.

$$
\begin{gathered}
p_{i}{ }^{\prime}=\alpha\left(p_{i}-p_{M}\right)+p_{M} \\
p_{i}{ }^{\prime}=-\beta\left(p_{M}-p_{\text {min }}\right)+p_{i}
\end{gathered}
$$

$\alpha$ : scaling factor for the part, $\beta$ : translation distance, $p_{M}$ : mean position of the part

$p^{\prime}$ : positions after deformation, $p_{i}:$ positions before deformation, $p_{\min }:$ chin position

It was anticipated that the parameters for the different features take different values, hence the personalized avatars based on the photo images were adapted again to the illustrated portraits using the developed avatar personalizing tool, an example is shown in Fig. 5. After the re-adaptation of the avatar shape, the scaling factors and the translation distances for each facial part were calculated for the seven portraits. The unique parameters for the five models and the average values for all seven models are shown in table 1.

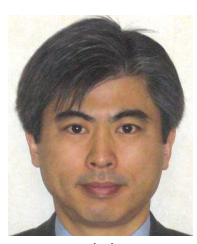

(a)

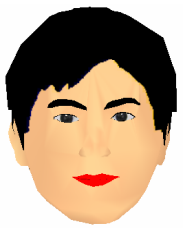

(b)

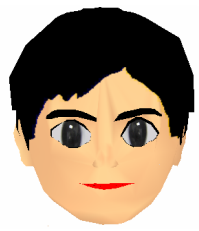

(c)

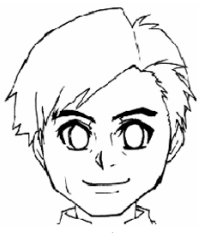

(d)

Fig. 5. Example of avatar personalization and deformation based on the photo image and the portrait. (a) Photo image of the model. (b) Personalized avatar based on the photo image. (c) Deformed avatar based on the portrait. (d) Portrait used for avatar deformation.

\begin{tabular}{|c|c|c|c|c|c|c|c|c|c|c|c|c|c|}
\hline \multirow{2}{*}{ Scale } & \multirow[t]{2}{*}{ Model } & \multicolumn{2}{|c|}{1} & \multicolumn{2}{|c|}{2} & \multicolumn{2}{|c|}{3} & \multicolumn{2}{|c|}{4} & \multicolumn{2}{|c|}{5} & \multicolumn{2}{|c|}{ average } \\
\hline & & $X$ & $\mathrm{Y}$ & $\mathrm{X}$ & $\mathrm{Y}$ & $X$ & $\bar{Y}$ & $\mathrm{X}$ & $\mathrm{Y}$ & $\mathrm{X}$ & $\mathrm{Y}$ & $\mathrm{X}$ & $\mathrm{Y}$ \\
\hline & eyebrow & 1.3 & 1.1 & 1.3 & 1.2 & 1.1 & 1.1 & 1.1 & 1.1 & 1.1 & 1.3 & 1.2 & 1.2 \\
\hline & Eye & 1.6 & 3.2 & 1.6 & 1.8 & 1.4 & 2.8 & 1.6 & 3.2 & 1.4 & 3.2 & 1.5 & 2.6 \\
\hline & Nose & 0.8 & 0.8 & 0.8 & 0.8 & 0.7 & 0.7 & 0.8 & 0.8 & 0.7 & 0.7 & 0.8 & 0.8 \\
\hline & Mouth & 1.2 & 0.4 & 1.0 & 0.5 & 0.9 & 0.3 & 1.0 & 0.4 & 0.9 & 0.5 & 1.0 & 0.4 \\
\hline \multirow{4}{*}{$\begin{array}{l}\text { trans- } \\
\text { lation }\end{array}$} & eyebrow & \multicolumn{2}{|c|}{0.1} & \multicolumn{2}{|c|}{0.1} & \multicolumn{2}{|c|}{0.2} & \multicolumn{2}{|c|}{0.1} & \multicolumn{2}{|c|}{0.2} & \multicolumn{2}{|c|}{0.1} \\
\hline & Eye & \multicolumn{2}{|c|}{0.2} & \multicolumn{2}{|c|}{0.1} & \multicolumn{2}{|c|}{0.3} & \multicolumn{2}{|c|}{0.3} & \multicolumn{2}{|c|}{0.3} & \multicolumn{2}{|c|}{0.2} \\
\hline & Nose & \multicolumn{2}{|c|}{0.2} & \multicolumn{2}{|c|}{0.1} & \multicolumn{2}{|c|}{0.3} & \multicolumn{2}{|c|}{0.4} & \multicolumn{2}{|c|}{0.4} & \multicolumn{2}{|c|}{0.3} \\
\hline & Mouth & \multicolumn{2}{|c|}{0.2} & \multicolumn{2}{|c|}{0.1} & \multicolumn{2}{|c|}{0.3} & \multicolumn{2}{|c|}{0.3} & \multicolumn{2}{|c|}{0.4} & \multicolumn{2}{|c|}{0.3} \\
\hline
\end{tabular}

Table 1. Unique and average deformation parameters 


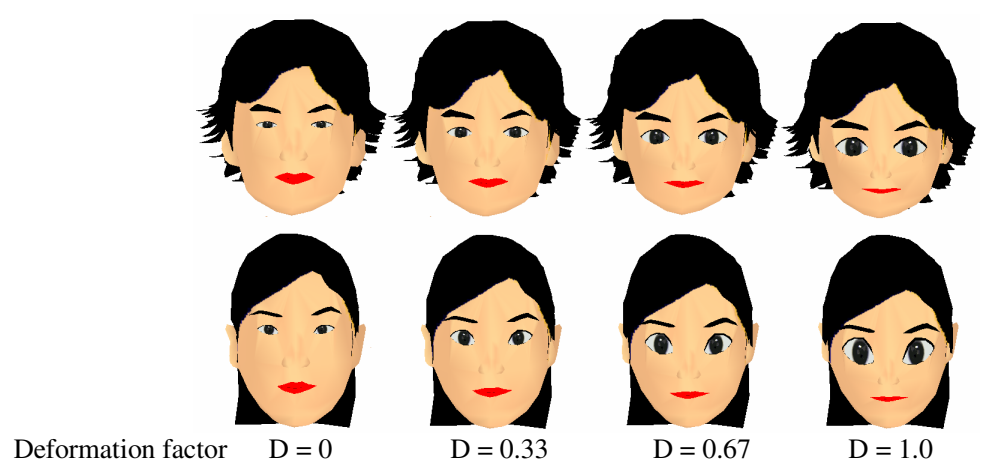

Fig. 6. Examples of automatically deformed avatars based on the extracted rule

The scaling factor for the eye vertical length is the largest in all the models, and some deviation is observed. The translation distance varies from 0.1 to 0.4 depending on the parts and the models. A similar tendency is observed among all the models.

Figure 6 shows examples of the deformed avatars obtained using the average deformation factors. The avatars were personalized first using photos, and deformed later. The left two figures are the personalized avatars. The right figures are those deformed according to the rule. The figures between them are also deformed avatars using scaled deformation factors that are $33 \%$ or $66 \%$ of the original factors. The automatic symmetry adjustment routine for eyes and eyebrows was implemented because the enlargement and the translation of the individual part emphasized the asymmetry of the facial features.

The upper avatars in Fig. 6 are for the model that was used for the deformation rule extraction; the lower avatar was not used. As the deformed avatar for the model independent of the rule extraction had no serious defects, it seems the extracted deformation rule can be generally applied.

\section{Subjective Evaluation of Avatars}

\subsection{Evaluation of the Avatars of Acquaintances}

Subjective evaluation of the avatars deformed based on the extracted rules was performed using the static frontal view of the avatars. The deformation factor was varied $0,33,66,100$, and $133 \%$, where $0 \%$ represents the personalized avatar and $100 \%$ represents the avatar deformed using the extracted rules. The similarity, favorability, and willingness to use it, were subjectively rated by 11 university students. The subjects evaluating the avatars were chosen from persons who know the models, to evaluate similarities between the model and avatar. The number of evaluated avatars was 11 , and the evaluation order was randomized.

The averages and the standard deviations for the avatars are shown in Fig. 7. It was confirmed that deformation improved the favorability scores as expected. The highest favorability was obtained at $66 \%$, and not $100 \%$. It appears that small shape defects were emphasized by the enlargement. The peak favorability scores of deformed avatars were higher than those of the non-deformed personalized avatars in all combinations of 


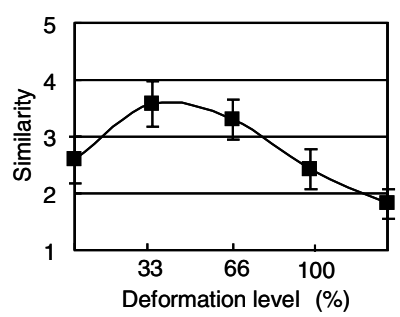

(a)

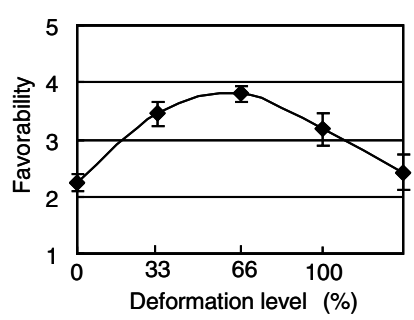

(b)

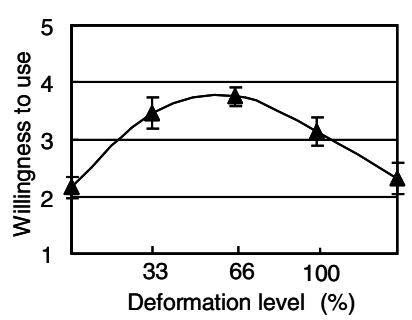

(c)

Fig. 7. Results of subjective evaluation of the avatars of acquaintances. (a) Similarity; (b) favorability; and (c) willingness to use scores.

evaluation subjects and model subjects. The results suggest that the existence of general deformation rules are independent of the models that improve favorability.

Interestingly, the highest similarity score was observed at 33\% deformation, not $0 \%$. This tendency coincides with the previous study that suggests that the subjective adaptation of the avatar shape resulted in larger eyes and mouth than their actual sizes [5]. It is speculated that the recognized size of the parts of the simple cartoonish avatar are smaller than the subjective size of photographic parts that have complicated textures.

The tendency of the willingness to use was similar to that of favorability. This demonstrated that the deformation of the personalized avatar provides more similarity and favorability, as well as the willingness to use it.

\subsection{Evaluation of the Avatars of Unknown Persons and Their Own Avatars}

To discuss the cross effect of similarity on favorability, a similar evaluation experiment on favorability was performed with eight subjects who do not know the models, the results of which are shown in Fig. 8. As seen in the figure, the tendencies were similar to those of the previous experiment. Therefore, the sense of similarity seems to have no affect on favorability.

To discuss the difference between the most favorable deformation level of their own avatar and the avatars of others, the most favorable deformation levels were investigated for nine modeled subjects. As shown in table 2, no difference was observed.

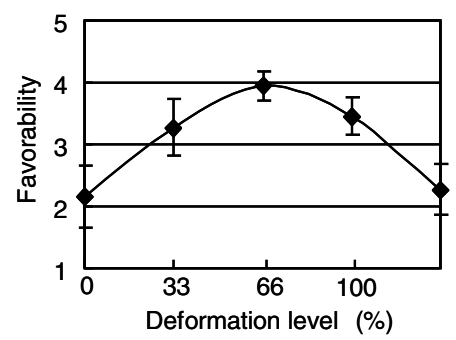

Fig. 8. Favorability scores of the avatars of unknown persons 
Table 2. Most favorable deformation levels of the avatars

\begin{tabular}{l|c|c|c|c|c|c|c|c|c}
\hline \multicolumn{1}{c|}{ subject } & $\mathrm{A}$ & $\mathrm{B}$ & $\mathrm{C}$ & $\mathrm{D}$ & $\mathrm{E}$ & $\mathrm{F}$ & $\mathrm{G}$ & $\mathrm{H}$ & $\mathrm{I}$ \\
\hline own & $66 \%$ & $66 \%$ & $66 \%$ & $100 \%$ & $66 \%$ & $66 \%$ & $33 \%$ & $66 \%$ & $66 \%$ \\
\hline others & $66 \%$ & $66 \%$ & $66 \%$ & $100 \%$ & $66 \%$ & $66 \%$ & $33 \%$ & $66 \%$ & $66 \%$ \\
\hline
\end{tabular}

\section{Discussion}

The most favored deformation level differed with the subject, but the peak favorability scores of the various deformed avatars were higher than those for the nondeformed personalized avatars for all combinations of evaluation subjects and models, including the modeled subjects who were not used for the deformation tendency analysis. This suggests the existence of a general deformation rule that improves favorability, independent of the target models. The applied deformation in this study was the enlargement and the downward translation of the facial features. That makes the facial proportions closer to those of children. A further improvement of the deformation rules could be discussed by analyzing the proportion differences between adults and children.

The most favored deformation level was lower than $100 \%$, which represents deformation by the extracted rule. One possible reason is the effect of the similarity impression; however, this is rejected because the evaluators who did now know the models had the same favored deformation levels. The most likely reason is that this is due to the effect of shape defects caused by the enlargement, which magnifies position errors and the polygon edges. Automatic adjustment of small shape defects, such as with smoothing, will be discussed.

The most favored deformation level of a person's own avatar was generally the same as that for avatars of others. It seems that none of the individuals had a problem in sending the most favorable avatar to a communication partner to represent him or her. However, individual differences were observed in determining the favorable deformation levels. Thus, it would be better to provide a function that allows users to interactively customize the deformation level of the displayed avatars.

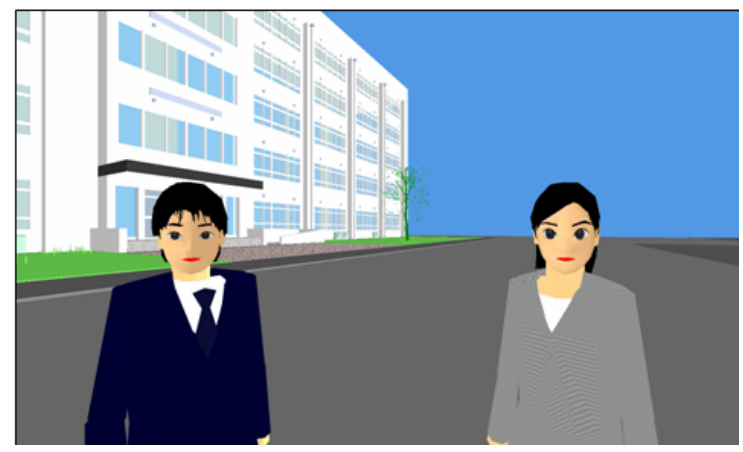

Fig. 9. Screenshot of the avatar-mediated voice chat system

In this study, we assumed that the compatibility between favorability and similarity provides more familiarity, and that higher familiarity provides real person-to-person 
communication between acquaintances. However, the evaluation was performed using the statically displayed avatars in this study. The effect of the deformed avatars on the communication impression is still unknown. The developed avatars are implemented in an avatar-mediated voice chat system [4], as shown in Fig. 9, and prepared for evaluation.

\section{Conclusions}

In this study, we focused on the positive impression of characters in Japanese cartoons. Deformation rules were extracted from cartoonish portraits and were implemented in an avatar personalizing tool. A subjective evaluation using the personalized and deformed avatars demonstrated the possibility of the avatars with similarity to the model, but without producing the "uncanny valley" sense. An evaluation in online communication is currently underway.

Acknowledgments. This work was partially supported by the MEXT Fund for Promoting Research on Symbiotic Information Technology.

\section{References}

1. Mehrabian, A.: Nonverbal communication. Aldine-Atherton (1972)

2. Mehrabian, A.: Silent messages. Wadsworth (1981)

3. Watanabe, T., Danbara, R., Okubo, M.: InterActor: Speech Driven Embodied Interactive Actor. In: 11th IEEE International Workshop on Robot and Human Interactive Communication, pp. 430-435 (2002)

4. Miyajima, T., Fujita, K.: Control of avatar's facial expression using fundamental frequency in multi-user voice chat system. In: Gratch, J., Young, M., Aylett, R.S., Ballin, D., Olivier, P. (eds.) IVA 2006. LNCS (LNAI), vol. 4133, p. 462. Springer, Heidelberg (2006)

5. Heike, M., Miyajima, T., Fujita, K.: Effect of personalized avatar on communication in virtual space voice chat system. In: Human Interface Symposium 2007 (2007) (in Japanese)

6. Mori, M.: The Uncanny Valley. Energy 7(4), 33-35 (1970) (translated by MacDorman, F.K., Minato, T.) 